\title{
Causes of Hypertension in the Young
}

\author{
BOON SENG OOI, ${ }^{*}$ M.B., M.R.A.C.P. ; BEATRICE T. M. CHEN, $\dagger$ M.B., M.R.A.C.P.
}

\author{
CHARLES C. S. TOH, $\ddagger$ M.B., M.R.C.P., F.A.C.C. ; OON TEIK KHOO, $\$ M.D., F.R.C.P.ED., F.R.C.P.GLASG.
}

\begin{abstract}
Cummary: Of 127 hypertensive patients aged 12 to 40 investigated by intravenous pyelography, abdominal aortography, and renal biopsy an underlying cause was found in $57 \%$. The proportion with secondary hypertension was higher in young patients and in those with severe hypertension. Primary arteritis of the aorta was an important cause of renovascular hypertension in an Asian population.
\end{abstract}

\section{Introduction}

Hypertension in the young has usually been attributed to some underlying cause, frequently renal. This view stems from the studies of Platt (1948), who found secondary hypertension in $75 \%$ of 64 hypertensive patients under the age of 40 . More recently, however, Breckenridge et al. (1967), using modern diagnostic techniques, were unable to confirm this finding. It is therefore of interest to compare our experience, using similar methods, in an unselected group of young hypertensive patients.

\section{Patients and Methods}

From March 1966 to October 1967139 patients aged 12 to 40 were seen in a medical unit in Singapore and diagnosed as having hypertension. A diastolic blood pressure of $100 \mathrm{~mm}$. $\mathrm{Hg}$ or more on at least two consecutive occasions was taken as showing hypertension. The patients were referred from outlying outpatient clinics, general practitioners, and the emergency unit of the General Hospital. Three medical units serve the General Hospital and the department of clinical medicine accepted patients purely on a rotational basis. Twelve of the patients died shortly after admission and have been excluded from this series. The patients were otherwise 1.. selected, and this is reflected in the proportion of those with mild hypertension. Referral selection was exercised in so 'ar as hypertension seen during pregnancy was managed by inother unit.

The patients were subjected to the following diagnostic procedures:

(1) Quantitative bacterial counts of fresh urine specimens.

(2) Estimation of 24-hour urine specimens for vanillylmandelic acid content.

(3) Intravenous pyelograms done with abdominal compression after 5 minutes. Films were taken after 5,10 , and 20 minutes. Rapid sequence pyelography with films at $1,2,3$, and 5 minutes was undertaken in patients in whom routine pyelography showed a disparity in kidney sizes of $1.5 \mathrm{~cm}$. or more.

(4) Renal arteriography performed by catheterizing the abdominal aorta via the femoral artery, the technique of Seldinger (1953) being used. Arteriography was performed on all consenting patients in whom pyelography had not shown the presence of cysts or definite chronic pyelonephritis.

(5) Percutaneous renal biopsy done on all consenting patients in whom intravenous pyelography had not revealed the presence of cysts, hydronephrosis, or unilateral non-functioning kidneys. No significant morbidity was encountered. Renal tissue obtained was

* Lecturer in Clinical Medicine.

+ Senior Registrar in Clinical Medicine.
$\ddagger$ Senior Lecturer.

S Professor.

Department of Clinical Medicine, University of Singapore. stained with haematoxylin and eosin, and the periodic-acid/Schiff and silver methenamine stains.

\section{Criteria for Diagnosis}

\section{Radiological Criteria}

Unilateral renal artery stenosis was considered significant if there was not only narrowing of a main vessel but also poststenotic dilatation, collateral vessel formation, and diminution in size of the affected kidney by $1.5 \mathrm{~cm}$. or more. Bilateral renal artery involvement was considered significant in all instances. Definite pyelonephritis was diagnosed when there was a renal cortical scar associated with abnormalities of the adjacent collecting system. Probable pyelonephritis was diagnosed if there was only a cortical scar.

\section{Histological Diagnosis}

This diagnosis has been based on established criteria (Heptinstall, 1966):

(a) Chronic glomerulonephritis was diagnosed when there was hyalinization of nearly all glomerular tufts with accompanying tubular, interstitial, and vascular changes.

(b) Chronic pyelonephritis was diagnosed when there was extensive infiltration of the interstitium with chronic inflammatory cells, tubular damage and atrophy, "tubular thyroidization," and varying degrees of glomerular damage, crowding, and periglomerular fibrosis.

(c) Focal nephritis was diagnosed when there was not only focal cellular proliferation but also cellular necrosis and nuclear fragmentation.

(d) Membranous glomerulonephritis was diagnosed when there was diffuse basement membrane thickening after staining with silver methenamine. Cellular proliferation was absent.

(e) Diffuse glomerulonephritis was diagnosed when there was diffuse proliferation of endothelial cells together with mesangial hypercellularity.

(f) Essential hypertension was diagnosed when renal histology was normal or showed hypertensive vascular changes only.

\section{Results}

The clinical presentation, the results of intravenous pyelography, abdominal arteriography, and renal biopsy, and the final diagnosis reached are summarized in Tables I to V.

The population comprised 98 Chinese, 14 Indians, and 15 Malays. Twenty-seven patients had a family history of hypertension. Of 127 pyelographic examinations $44 \%$ showed abnormalities. Of interest were 10 patients with differences of

TABle I.-Clinical Data of 127 Patients

\begin{tabular}{|c|c|c|c|c|c|c|c|c|c|c|}
\hline \multicolumn{9}{|c|}{ Age in Years } & \multirow{2}{*}{$\begin{array}{c}\text { Male } \\
9 \\
6 \\
16 \\
21 \\
43\end{array}$} & \multirow{2}{*}{$\begin{array}{c}\text { Female } \\
4 \\
2 \\
3 \\
10 \\
13\end{array}$} \\
\hline $\begin{array}{l}<20 \ldots \\
20-24 \\
25-29 \\
30-34 \\
35-40\end{array}$ & $\begin{array}{l}\cdots \\
\cdots \\
\cdots \\
\cdots\end{array}$ & $\begin{array}{l}\cdots \\
\cdots \\
\cdots \\
\cdots\end{array}$ & $\begin{array}{l}\cdots \\
\cdots \\
\cdots \\
\cdots\end{array}$ & $\begin{array}{l}\cdots \\
\cdots \\
\cdots \\
\cdots\end{array}$ & $\begin{array}{l}\cdots \\
\cdots \\
\cdots \\
\cdots\end{array}$ & $\begin{array}{l}\cdots \\
\cdots \\
\cdots \\
\cdots\end{array}$ & $\begin{array}{l}\cdots \\
\cdots \\
\cdots \\
\cdots\end{array}$ & $\begin{array}{l}\cdots \\
\cdots \\
\therefore \\
\therefore\end{array}$ & & \\
\hline & & & & & & & & & 95 & 32 \\
\hline
\end{tabular}

Fundal grading of hypertension (Keith, Wagener, and Barker, 1939): grade I, 71 ; 
Table II.-Results of Intravenous Pyelography in 127 Patients

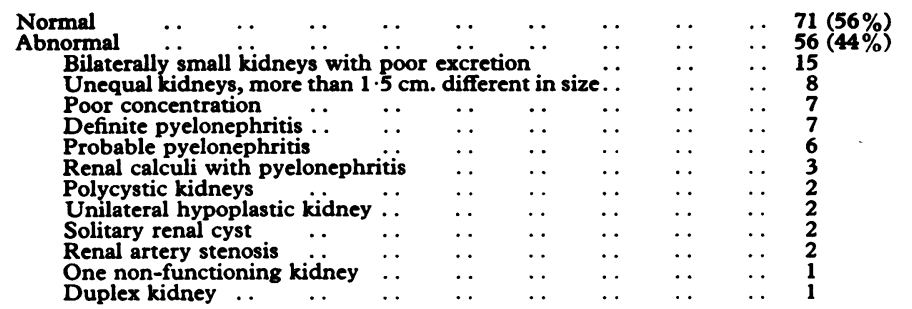

TABLE III.-Incidence of Aortic and Renovascular Abnormalities in 90 Abdominal Arteriograms

Bilateral renal arterial occlusion

Left renal artery stenosis

Right renal artery stenosis

Occlusion of descending aorta and subclavian artery

Thrombosis of descending aorta at level of renal arteries

Non-significant renal artery stenosis

TABLE IV.-Renal Histological Findings in 57 Patients

Normal or hypertensive changes only

ther renal disease ... chephritis

Chronic glomeruloneph

Focal glomerulonephritis

Diffuse proliferative glomerulonephritis

Membranous glomerulonephritis

Table V.-Final Diagnoses in 127 Patients

Essential hypertension

Secondary hypertension

Chronic glomeruloneph

Chronic pyelonephritis with calculus

Renal artery stenosis

Proliferative glomerulonephritis

Focal glomerulonephritis

Membranous glomerulonephritis

Solitary renal cyst

Unilateral hypoplastic kidney

Polycystic kidneys

Unilateral non-functioning kidney

Duplex kidneys

kidney sizes by $1.5 \mathrm{~cm}$. Two of these could be diagnosed with some certainty by rapid sequence pyelography as having renal artery stenosis. This diagnosis was confirmed later by arteriography. The other eight had normal arteriograms. Renal biopsy of the smaller kidney in two of these patients

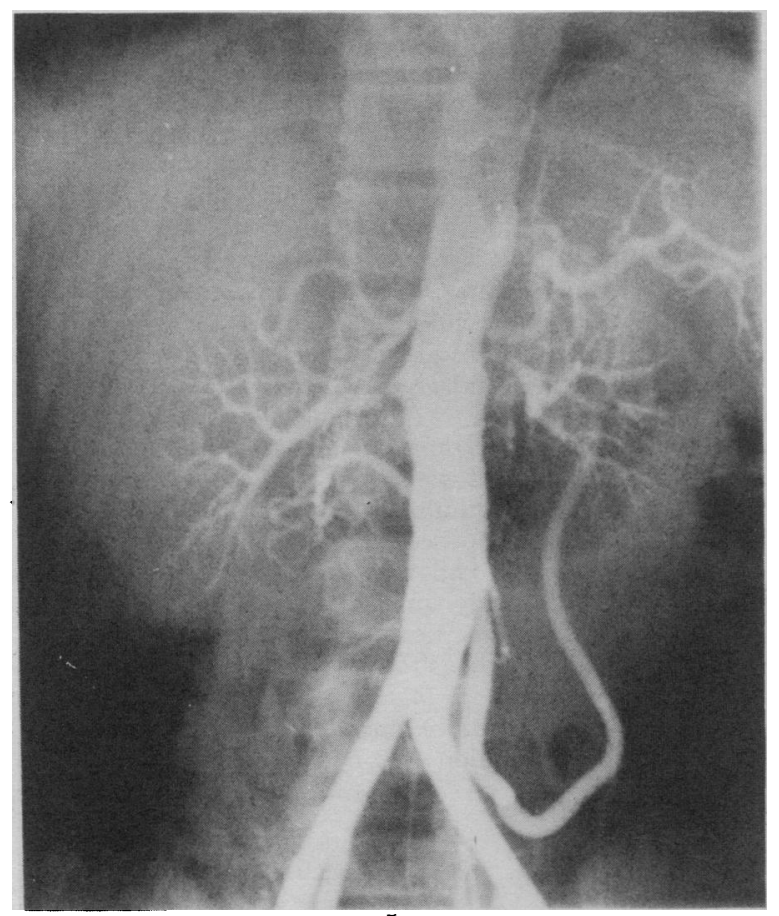

Abdominal sortogram of a patient with arteritis of the aorta. Note the irregularities in the wall of the aorta and the presence of bilateral renal artery stenosis. showed only hypertensive changes. The significance of this disparity in kidney size is not apparent. Only 12 patients were found by arteriography to have significant renal arterial stenosis or occlusion. Definite evidence of arteritis in other parts of the arterial circulation was evident in five of them: the accompanying aortogram is an instance of this. Renal biopsy allowed the diagnosis of primary renal disease in $51 \%$ of 57 biopsies.

The relationship between age, fundal grading of hypertension, and the proportion of patients with secondary hypertension is shown in Tables VI and VII. It supports the

TABLE VI.-Relation between Age and Incidence of Secondary Hypertension

\begin{tabular}{|c|c|c|c|c|c|}
\hline $\begin{array}{l}\text { Age in years } \\
\text { Percentage of patients } \\
\text { hypertension } \ldots\end{array}$ & $\begin{array}{l}<20 \\
85\end{array}$ & $\begin{array}{c}20-24 \\
75\end{array}$ & $\begin{array}{c}25-29 \\
63\end{array}$ & $\begin{array}{c}30-34 \\
70\end{array}$ & $\begin{array}{c}35-4 c \\
38\end{array}$ \\
\hline
\end{tabular}

TABLE VII.-Relation between Fundal Grading of Hypertension and Incidence of Secondary Hypertension

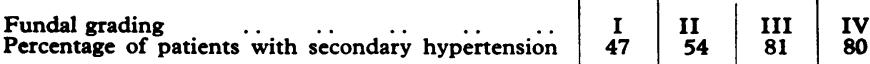

contention that the incidence of secondary hypertension in a population is a function of the age structure and the degree of severity of hypertension in that population.

\section{Discussion}

There are two comparable series with which to evaluate the significance of a finding of $57 \%$ of cases of secondary hypertension in a young population. Platt (1948) was able to detect a cause in $75 \%$ of 64 patients. By contrast, Breckenridge et al. (1967), using similar techniques to the present study, except renal biopsy, described a cause in only $32 \%$ of 229 patients. The high proportion of secondary hypertension diagnosed in Platt's series is probably accounted for by the number of patients with severe hypertension, 51 out of 64 patients having a diastolic blood pressure of $130 \mathrm{~mm}$. $\mathrm{Hg}$ or more. Of our 31 patients with hypertension of fundal grades III and IV, a primary pathology was found in $80 \%$. The age of onset is probably also important. Only one of Platt's patients was over the age of 35 . Secondary hypertension was diagnosed in $70 \%$ of our 71 patients under 35 years. The differences between our series and those reported by Breckenridge et al. (1967) are not so readily: apparent. Both hypertensive populations are comparable with respect to severity of hypertension. The age distribution is not available for comparison. Another factor is the frequent use of renal biopsy in this study. In fact, in the absence of renal histological findings, only $40 \%$ of patients would have a detectable cause. In terms of the causes of hypertension, the preponderance of glomerulonephritis over pyelonephritis as a cause in this series might be accounted for by the proportionately smaller number of female patients seen.

Renovascular hypertension was present in only $9 \%$ of the patients. Poutasse and Dustan (1957) found significant renal artery stenosis in $30(29 \%)$ of a selected series of 104 hypertensive patients. Similar results have been obtained in another selected series (Fenton et al., 1966). By contrast, in an unselected series of patients (Breckenridge et al., 1967) only $5 \%$ had renovascular hypertension. Of more interest in our series is the number with bilateral renal arterial involvement. Pyelographic examination would be of little help as a screening procedure for renovascular hypertension in such instances. The presence of arteritis, probably primary in aetiology, accounted for the large proportion with bilateral renal artery disease. The presence of arteritis as a cause of renal hypertension has been documented by Danaraj and Ong (1959), and our study shows its importance as a cause of renovascular hypertension in an Asian population.

Renal biopsy has been found to be a useful diagnostic tool 
in the elucidation of primary morbidity. The differentiation between changes due to nephrosclerosis, chronic nephritis, and chronic pyelonephritis can be difficult, and the final diagnosis has been reached on correlations between histological, radiological, and bacteriological findings, together with the clinical picture. Focal nephritis has been associated with hypertension (Kincaid-Smith, 1966). The exact pathophysiological significance of this is not clear, though a familial type with dominant inheritance associated with severe hypertension has been described. Finally, this study emphasizes the need to investigate young hypertensive patients, especially those who are under the age of 35 or who have fundal grades III and IV hypertension, as a large proportion of these will be found to have an underlying cause.
We thank Dr. Tan Kheng Khoo, of the department of pathology of Outram Road General Hospital, Singapore, for his help in interpreting the renal histology, and the radiologists of Outram Road General Hospital, especially Dr. S. F. Yu, for their generous co-operation in the radiological examinations done in this study.

Breckenridge, A., Preger, L., Reflery, C. T., and Laws, J. W. (1967) Quarterly fournal of Medicine, 36, 549 .

Danaraj, T. J., and Ong, W. H. (1959). Circulation, 20, 856.

Fenton, S. S. A., Lyttle, J. A., and Pantridge, J. F. (1966). Lancet, 2, 117. Heptinstall, R. H. (1966). Pathology of the Kidney. London, Churchill. Keith, N. M., Wagener, H. P., and Barker, N. W. (1939). Americal fourna of the Medical Sciences, $197,332$.

Kincaid-Smith, P. (1966). In Antihypertensive Therapy, ed. F. Gross, p. 485. Berlin, Springer.

Platt, R. (1948). Quarterly fournal of Medicine, 17, 82.

Poutasse, E. F., and Dustan, H. P. (1957). Fournal of the American Medical Association, 165, 1521 .

Seldinger, S. I. (1953). Acta Radiologica, 39, 368.

\title{
Reversible Cerebral Oedema Complicating Diabetic Ketoacidosis
}

\author{
ALLAN L. METZGER,* M.D. ; ARTHUR H. RUBENSTEIN, $†$ M.B., B.CH., M.R.C.P.
}

\begin{abstract}
Cummary: A young man with diabetic ketoacidosis developed the clinical features of cerebral oedema. This resolved without specific treatment. The mortality from ketoacidosis might be reduced if the syndrome was suspected and treated earlier.
\end{abstract}

\section{Introduction}

The mortality from diabetic ketoacidosis varies between 8 and $16 \%$, depending on the age of the population studied (Greenway and Read, 1958; Fitzgerald et al., 1961; Young and Bradley, 1967). At necropsy many of these patients show the pathological characteristics of cerebral oedema. This syndrome, however, has seldom been considered or diagnosed by the clinician, and the pertinent literature deals mainly with its pathogenesis and irreversible course. We wish to draw attention to a patient with this condition who recovered spontaneously. Retrospective consideration of the clinical progress of other patients with diabetic ketoacidosis leads us to believe that this syndrome may not be very uncommon.

\section{Case Report}

An 18-year-old negro was brought to Billings Hospital emergency room moderately confused and lethargic. His wife stated that until two weeks previously he had been in excellent health. He then developed weakness, malaise, weight loss, shortness of breath, poly uria, and polydipsia. He complained of abdominal pain, nausea, and recurrent vomiting. There was no history of trauma or infection. His maternal grandmother had diabetes mellitus requiring insulin.

On physical examination he was lethargic, thin, and profoundly weak, with pronounced Kussmaul respiration. His pulse was $136 /$ minute, blood pressure $115 / 70$, respiration $36 /$ minute, and rectal temperature $37^{\circ} \mathrm{C}$. He was dehydrated, and acetone was noted in his breath.

Initial laboratory data included a blood sugar of $765 \mathrm{mg}$. and blood urea nitrogen of $22 \mathrm{mg}$. $/ 100 \mathrm{ml}$; the serum sodium was 130 $\mathrm{mEq}$, potassium $5.2 \mathrm{mEq}$, chloride $92 \mathrm{mEq}$, and bicarbonate less than $10 \mathrm{mEq} / \mathrm{l}$. The calculated serum osmolality was 328 mOsm/l. (Dillon et al., 1936). Serum acetone was 3+ at 1:2 dilution with Acetest tablets. Arterial blood gases were $\mathrm{CO}_{2} 12 \mathrm{~mm}$.

- Resident in Medicine.

$\dagger$ Associate Professor of Medicine.

University of Chicago, Chicago, Illinois. and $\mathrm{O}_{2} 118 \mathrm{~mm}$. $\mathrm{Hg}$; the $p \mathrm{H}$ was $7 \cdot 25$. The packed cell volume was $51 \%$ and the white blood count 21,500 cells/cu.mm., with $95 \%$ polymorphonuclear leucocytes.

Intravenous therapy was initiated with 1 litre of $0.45 \%$ saline to which $44 \mathrm{mEq}$ of sodium as bicarbonate was added; concomitantly, 100 units of regular insulin was injected subcutaneously. Over the first four hours he received 3 litres of $0.45 \%$ saline and $88 \mathrm{mEq}$ of sodium bicarbonate. The blood sugar fell to $376 \mathrm{mg} . / 100 \mathrm{ml}$., serum acetone decreased to $1+$ without dilution, and the serum bicarbonate rose to $10 \mathrm{mEq} / 1$. Serum sodium, potassium, and chloride levels approached normal values. At this time he was alert, but complained of nausea and vomited. During the next four hours 50 units of regular insulin, 2.5 litres of $5 \%$ dextrose in $0.33 \%$ saline, $88 \mathrm{mEq}$ of sodium bicarbonate, and 80 $\mathrm{mEq}$ of potassium chloride were given. The blood sugar fell to $240 \mathrm{mg}$. $/ 100 \mathrm{ml}$., serum bicarbonate increased to $21 \mathrm{mEq} / 1$., and serum acetone was negative. His mental status improved noticeably and headache, nausea, and vomiting abated. He remained afebrile and had a satisfactory urinary output.

About 10 hours after admission his mental status suddenly worsened despite near normal serum electrolyte (sodium $133 \mathrm{mEq}$, potassium $3.7 \mathrm{mEq}$, chloride $101 \mathrm{mEq}$, bicarbonate $21 \mathrm{mEq} / 1$.) and blood glucose values $(200 \mathrm{mg} . / 100 \mathrm{ml}$.). The serum osmolality was $295 \mathrm{mOsm} / 1$. He complained of headache and became lethargic, confused, and difficult to arouse. Moderate neck stiffness was elicited, but neither papilloedema nor localizing neurological signs were observed. Lumbar puncture revealed an opening pressure of $420 \mathrm{~mm}$. and a closing pressure of $410 \mathrm{~mm}$.; there were no cells, and all cultures were subsequently negative. The cerebrospinal fluid protein was $25 \mathrm{mg}$. and glucose $270 \mathrm{mg}$. $/ 100 \mathrm{ml}$. The simultaneous blood sugar value was $176 \mathrm{mg} . / 100 \mathrm{ml}$. A diagnosis of cerebral oedema was made, and though treatment with mannitol and steroids was considered we decided to observe his course for a short time before their administration. Gradually over the next four hours the patient improved: he became less irritable, was more alert, and began to converse rationally and spontaneously. Thereafter he showed steady improvement, and some eight hours after the lumbar puncture his mental status returned to normal. The remainder of his stay in hospital was uneventful, and after two weeks he was discharged on 24 units of insulin injection and 24 units of protamine zinc insulin injection daily. At later outpatient visits neurological examination was negative and his mental status was normal.

\section{Discussion}

This report illustrates the occurrence of cerebral oedema complicating the management of moderately severe diabetic ketoacidosis. The clinical picture of mental deterioration 